Revista Costarricense de Psicología, Vol. 29, n. ${ }^{\circ} 43,2010,35-46$.

DOI: http://dx.d

35

\title{
Control del estrés, ira y ansiedad en pacientes con hipertensión arterial mediante TREC
}

\section{Stress, Anger, and Anxiety Management through REBT in Patients with Arterial Hypertension}

\author{
Federico Holst Schumacher ${ }^{\mathrm{a}}$, Diego Quirós Morales ${ }^{\mathrm{b}}$ * \\ Universidad de Iberoamérica, Costa Rica \\ (recibido 11 de enero 2010; aceptado 15 de enero de 2011)
}

\begin{abstract}
Resumen
La hipertensión Arterial constituye uno de los trastornos de mayor incidencia a nivel internacional, se recomienda el manejo de los factores psicosociales que influyen en la enfermedad. Se valoró la implementación de la Terapia Racional Emotiva Conductual (TREC) sobre los niveles de hipertensión arterial, estrés, ira y ansiedad en cuatro sujetos hipertensos de la comunidad de San Miguel de Sarapiquí. Se presentó una disminución en los niveles de tensión arterial, estrés, ira y ansiedad posterior a la aplicación de la TREC en los pacientes hipertensos.
\end{abstract}

Palabras clave: hipertensión arterial, estrés, ira, ansiedad, terapia racional emotiva conductual

\begin{abstract}
Hypertension is one of the disorders with the highest incidence worldwide; it is recommended that the psychosocial factors that influence it be managed. In this project we evaluated the implementation of Rational Emotive Behavior Therapy (REBT) on the levels of hypertension, stress, anger, and anxiety in four hypertensive subjects from the community of San Miguel de Sarapiqui, in Costa Rica. There was a decrease in blood pressure levels, stress, anger, and anxiety after REBT was applied for the hypertensive patients.
\end{abstract}

Keywords: hypertension, stress, anger, anxiety, Rational Emotive Behavior Therapy

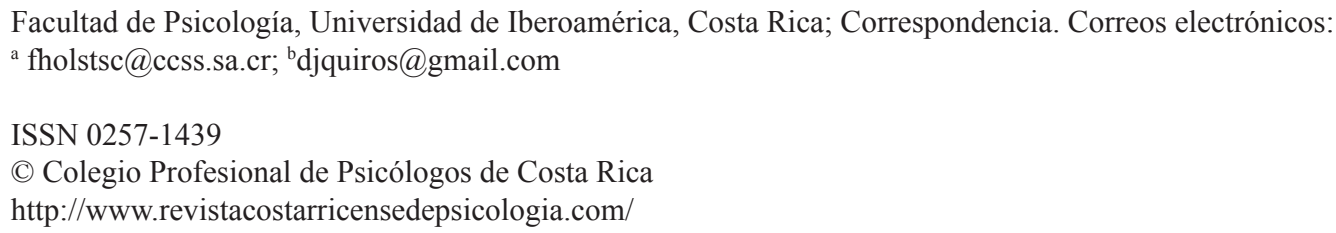


La hipertensión arterial es una enfermedad crónica, controlable, de etiología multifactorial, caracterizada por aumento sostenido de las cifras de presión arterial (presión sistólica igual o mayor a $140 \mathrm{mmHg}$ o presión diastólica igual o mayor a $90 \mathrm{mmHg}$ (Chapuis, Barriguete, Cheron, Hernández, Vieyra, \& García, 2006).

Esta enfermedad constituye uno de los trastornos de mayor incidencia a nivel internacional y es reconocida entre los principales factores de riesgo de los trastornos cardiovasculares y cerebrovasculares, que se ubican entre las primeras causas de morbimortalidad a nivel mundial (Molerio \& García, 2004a).

El impacto económico, social y en salud que tiene esta enfermedad es de grandes proporciones; por lo tanto, es importante que se realicen esfuerzos para su detección temprana, diagnóstico correcto y tratamiento adecuado por parte de las autoridades correspondientes. Dentro de los factores etiológicos reconocidos en la hipertensión arterial se encuentra el componente psicosocial como responsables o bien agravante de la enfermedad. Asimismo, los avances en la investigación actual y los rápidos progresos tecnológicos en diversas áreas han perfilado de forma progresiva y clara la importancia de ciertos comportamientos como agentes responsables mediadores en la salud y la enfermedad (Miguel-Tobal, Cano-Vindel, Casado-Morales, \& Escalona-Martínez, 1994).

La multicausalidad se centra en considerar simultáneamente factores genéticos, ambientales, psicofisiológicos y su interacción entre sí, como elemento predisponente para que la hipertensión arterial se presente en un determinado individuo (Miguel-Tobal et al., 1994).

Dentro de los factores de riesgo modificables, se encuentran la Diabetes Mellitus, la hipercolesterolemia (niveles mayores a $200 \mathrm{mg} / \mathrm{dl}$ ), hipertrigliceridemia (niveles mayores a $150 \mathrm{mg} / \mathrm{dl}$ ), hiperuricemia, tabaquismo, sobrepeso (un índice de masa corporal mayor a 25), y sedentarismo (Chapuis et al., 2006). También, se identifica al exceso de ingesta de sodio y exceso en el consumo de alcohol como factores de riesgo modificables (Jiménez, 2002).

Los factores de riesgo no modificables son la edad (mayores de 45 años), sexo "masculino", raza "negra", y antecedente en familiar directo de hipertensión arterial, Diabetes Mellitus, cardiopatía coronaria o enfermedad vascular cerebral (Chapuis et. al., 2006).

La Caja Costarricense de Seguro Social (CCSS) identifica como factores de riesgo mayores al tabaquismo, dislipidemia, Diabetes Mellitus, edad mayor a 60 años, sexo masculino y mujeres postmenopáusicas, y la historia de infarto al miocardio o muerte por enfermedad del corazón en un familiar en primer grado: mujeres menores a 65 años y hombres menores de 55 años (Jiménez, 2002).

Además, dentro de las medidas no farmacológicas recomendadas por parte de la CCSS se incluyen: reducir el peso, evitar el consumo excesivo de alcohol y sal, realizar actividad física, eliminar el tabaquismo, cambios en la alimentación, y control de estrés (Jiménez, 2002). Desde hace años, las organizaciones sanitarias internacionales han sugerido la importancia de promover estrategias cognitivas y de comportamiento, para facilitar el control de la presión arterial (Labiano, Brusasca, \& Gargani, 2005).

En la mayoría de las ocasiones, los pacientes hipertensos optan, en primera instancia, por un tratamiento farmacológico. Aun así, se ha reportado que el $70 \%$ de los pacientes en tratamiento antihipertensivo, no controla adecuadamente sus cifras de tensión arterial (Fernández, Martín, \& Domínguez, 2003). Se afirma que la baja adherencia a las medidas terapéuticas se encuentra entre las principales causas de esta situación. Estos mismos autores señalan que entre el 29\% y el 56\% de los pacientes hipertensos no toman la medicación de forma indicada. En general, el grado de 
falta de cumplimiento es aproximadamente del 50\% (Brannon \& Feist, 2001), DiMatteo (2004) habla de un $45 \%$ de incumplimiento en tratamientos a largo plazo, como en las enfermedades crónicas.

Ante esta problemática se recomienda, entre otras estrategias de acción multidisciplinarias para la prevención, control y tratamiento adecuados de la hipertensión arterial en la población, el manejo de los factores psicosociales que influyen en la enfermedad. Se ha demostrado que el estrés y las reacciones emocionales como la ansiedad, la ira o la tristeza presentan correlatos fisiológicos que afectan la presión arterial, y por lo tanto la hipertensión arterial es considerada hoy como un trastorno psicofisiológico (Molerio \& García, 2004b), aun cuando no exista evidencia suficiente para fundamentar dicha aseveración (Friedman, Schwartz, Schall, Pieper, Gerin, \& Pickering, 2001).

Se han realizado estudios donde se ha evidenciado la relación entre el componente conductual y la hipertensión arterial. Sommers y Greenberg (1989) encontraron que los individuos hipertensos presentaban dificultades con la expresión de la cólera y mayor ansiedad interpersonal, así como un uso más frecuente de mecanismos defensivos con respecto a sus experiencias emocionales. Por su parte, Jorgensen, Johnson, Kolodziej y Schreer (1996) han señalado que en los hipertensos se da una mayor emocionalidad negativa y defensividad y menor expresión del afecto. Vögele y Steptoe (1992) mencionan niveles más elevados de cólera contenida o encubierta como rasgos presentes en los hipertensos.

Es claro que las emociones y el estrés tienen una importante función adaptativa. Sin embargo, Molerio y García (2004a), afirman que bajo determinadas condiciones, el estrés puede generar estados emocionales negativos como la ansiedad y la depresión, que pueden tener un papel desencadenante o agravante en determinados trastornos como la hipertensión arterial esencial, por tanto se vuelve relevante el entrenamiento adecuado para su control. Las técnicas y modelos cognitivo-conductuales de terapia y modificación de conducta son muy utilizados para complementarse, junto con el abordaje médico, en el tratamiento de múltiples enfermedades, entre ellas la hipertensión arterial (Miguel-Tobal et al., 1994).

\section{Pregunta de investigación}

Esta investigación busca demostrar la eficacia de la TREC en la disminución de los niveles de estrés, ansiedad, ira y presión arterial en pacientes hipertensos.

El impacto social que estas medidas podrían tener en el sistema de salud de nuestro país sería importante, siempre y cuando se logre que los pacientes comprendan que ellos mismos son los que tienen la mayor responsabilidad en el momento de controlar todos los factores que se conoce elevan la presión arterial.

Se considera que en la medida que el Sistema de Salud le dé más importancia a las medidas no farmacológicas en los programas de control de enfermedades crónicas como la hipertensión arterial, podría reducirse la inversión económica que se realiza a nivel nacional en la compra y despacho de medicamentos antihipertensivos, asegurando el control de los pacientes. 


\section{Método}

\section{Tipo de estudio}

Se realizó un estudio cuasi-experimental con un diseño pre-pos de un solo grupo (Hernández, Fernández, \& Baptista, 2006)

\section{Participantes}

Se trabajó con un total de cuatro participantes, todos hipertensos de los Equipos Básicos de Atención Integral en Salud (EBAIS) de San Miguel. Estos pacientes se escogieron al azar entre la lista de hipertensos con riesgos A o B aportada por los funcionarios de dicho EBAIS. Los cuatro participantes fueron renombrados por razones de confidencialidad y clasificación en función de la estratificación del riesgo y tratamiento de la hipertensión arterial según la Caja Costarricense de Seguro Social (CCSS). La tabla 1 expresa el pseudónimo y clasificación de cada participante.

Tabla 1

Pseudónimo y clasificación empleada para cada paciente

\begin{tabular}{ccc}
\hline Número de Paciente & Pseudónimo & Clasificación \\
\hline 1 & A.J. & $2 \mathrm{~B}$ \\
2 & M.P. & $2 \mathrm{~B}$ \\
3 & S.M. & $1 \mathrm{~A}$ \\
4 & M.S. & $1 \mathrm{~B}$ \\
\hline
\end{tabular}

Criterios de inclusión:

- Pacientes hipertensos con más de tres años de tener el diagnóstico.

- Clasificación de riesgo grados A o B (sin factores de riesgo o con factores de riesgo menores, respectivamente).

- Pacientes mayores de edad, que refieren su consentimiento al estudio.

- Pacientes que sepan leer y escribir.

- Pacientes de cualquier sexo.

- Pacientes que tengan más de tres años de vivir en San Miguel de Sarapiquí, Costa Rica.

- Pacientes que se encuentren en control farmacológico en la Clínica local de la CCSS para el control de la hipertensión arterial, y que refieran ser adherentes al tratamiento de una forma continua (y que no hayan tenido modificaciones en su tratamiento en los últimos tres meses).

Criterios de exclusión:

- Pacientes hipertensos con clasificación de riesgo C (diabéticos o con lesión de órgano blanco).

- Pacientes que hayan estado o estén en control psicológico de algún tipo, o se encuentren en estado psicótico en el momento de la investigación (para garantizar la confiabilidad de los resultados).

- Pacientes que se encuentren tomando medicamentos antidepresivos o ansiolíticos al momento de la investigación. 
- Pacientes que se encuentren empezando algún tipo de tratamiento alternativo para la tensión arterial (por ejemplo, acupuntura u homeopatía), o que estén empezando a realizar cambios hacia estilos de vida saludable (por ejemplo, empezando con algún programa de ejercicios o dieta).

- Pacientes manifiestamente no cooperadores o con incapacidad para entender o llenar los cuestionarios.

Para la estratificación de riesgo, se toman como referencia los parámetros establecidos por la CCSS (ver tabla 2).

Tabla 2

Estratificación del riesgo y tratamiento de la hipertensión arterial según la Caja Costarricense de Seguro Social

\begin{tabular}{|c|c|c|c|}
\hline $\begin{array}{l}\text { Categoría de la presión } \\
\text { arterial }\end{array}$ & $\begin{array}{l}\text { Grupo de Riesgo A } \\
\text { (sin factores de riesgo } \\
\text { mayores o ECV) }\end{array}$ & $\begin{array}{l}\text { Grupo de Riesgo B } \\
\text { (al menos un factor } \\
\text { de riesgo, excluye } \\
\text { Diabetes, ausencia de } \\
\text { ECV) }\end{array}$ & $\begin{array}{l}\text { Grupo de Riesgo C (ECV } \\
\text { y/o diabetes con o sin } \\
\text { otros factores de riesgo) }\end{array}$ \\
\hline $\begin{array}{l}\text { Alta "Normal" } \\
(130-139 / 85-89)\end{array}$ & $\begin{array}{l}\text { Tratamiento no } \\
\text { farmacológico }\end{array}$ & $\begin{array}{l}\text { Tratamiento no } \\
\text { farmacológico }\end{array}$ & $\begin{array}{l}\text { Tratamiento no } \\
\text { farmacológico y } \\
\text { farmacológico }\end{array}$ \\
\hline $\begin{array}{l}\text { Grado } 1 \text { “Óptima” } \\
(140-159 / 90-99)\end{array}$ & $\begin{array}{l}\text { Tratamiento no } \\
\text { farmacológico }\end{array}$ & $\begin{array}{l}\text { Tratamiento no } \\
\text { farmacológico }\end{array}$ & $\begin{array}{l}\text { Tratamiento no } \\
\text { farmacológico y } \\
\text { farmacológico }\end{array}$ \\
\hline $\begin{array}{l}\text { Grados } 2 \text { y } 3 \\
(160 / 100 \text { o más) }\end{array}$ & $\begin{array}{l}\text { Tratamiento no } \\
\text { farmacológico y } \\
\text { farmacológico }\end{array}$ & $\begin{array}{l}\text { Tratamiento no } \\
\text { farmacológico y } \\
\text { farmacológico }\end{array}$ & $\begin{array}{l}\text { Tratamiento no } \\
\text { farmacológico y } \\
\text { farmacológico }\end{array}$ \\
\hline
\end{tabular}

Nota $: \mathrm{ECV}=$ enfermedades cerebro vasculares. Fuente: Jiménez (2002).

\section{Diseño}

El presente estudio se desarrolló bajo un diseño cuasi-experimental empleando un solo grupo, valorado antes y después de la aplicación del tratamiento con el fin de evaluar los cambios producidos sobre este. Las variables contempladas para el diseño fueron:

Variables dependientes: presión arterial, nivel de estrés percibido, nivel de ansiedad.

Variable independiente: Terapia Racional Emotivo Conductual

\section{Procedimiento}

Se realizaron ocho sesiones de trabajo, tanto individuales como grupales (ver tabla 3). En la primera sesión se valoraron los niveles de tensión arterial (por medio de esfigmomanómetro manual) ansiedad, ira y estrés de los sujetos en la primera sesión. Posteriormente se trabajó en función de los resultados obtenidos en dicha valoración durante seis sesiones, una vez por semana con una 
duración promedio de 90 minutos, al usar técnicas de Terapia Racional Emotiva Conductual. En la sesión final, se repitieron las evaluaciones realizadas en la primera sesión.

Tabla 3

Descripción de objetivos y actividades desarrollados en las sesiones de trabajo

\begin{tabular}{|c|c|c|c|}
\hline $\begin{array}{l}\text { Número } \\
\text { de Sesión }\end{array}$ & $\begin{array}{l}\text { Tipo de } \\
\text { Sesión }\end{array}$ & Objetivo de la sesión & Actividades realizadas \\
\hline 1 & Grupal & $\begin{array}{l}\text { Encuadre general sobre } \\
\text { proceso a iniciar, además } \\
\text { de realizar las mediciones } \\
\text { físicas y psicométricas. }\end{array}$ & $\begin{array}{l}\text { 1. Exposición de los objetivos del proceso } \\
\text { 2. Toma de tensión arterial } \\
\text { 3. Aplicación de: } \\
\text { - Cuestionario de Estrés Percibido } \\
\text { - Inventario de Hostilidad de Buss-Durkee } \\
\text { - Inventario de Ansiedad Rasgo-Estado }\end{array}$ \\
\hline 2 & Grupal & $\begin{array}{l}\text { Exposición del modelo } \\
\text { ABC de la Terapia Racional } \\
\text { Emotivo Conductual } \\
\text { (TREC). }\end{array}$ & $\begin{array}{l}\text { 1. Exposición del Modelo ABC de la TREC } \\
\text { 2. Explicación de los tres tipos de } \\
\text { creencias evaluadoras que resultan ser } \\
\text { desadaptativas y disfucionales: } \\
\text { - Tremendismo, } \\
\text { - Condenación y } \\
\text { - Baja Tolerancia a la Frustración }\end{array}$ \\
\hline 3 & Grupal & $\begin{array}{l}\text { Exposición de las técnicas } \\
\text { de debate y tareas para la } \\
\text { casa. }\end{array}$ & $\begin{array}{l}\text { 1. Exposición del debate y tareas casa. } \\
\text { 2. Práctica grupal de autodebate. } \\
\text { 3. Tareas para la casa }\end{array}$ \\
\hline 4 & Individual & $\begin{array}{l}\text { Exposición de resultados } \\
\text { obtenidos con la valoración } \\
\text { realizada en la primera } \\
\text { sesión e inicio de la TREC. }\end{array}$ & $\begin{array}{l}\text { 1. Revisión de la tarea para la casa. } \\
\text { 2. Exposición de los resultados de las } \\
\text { pruebas aplicadas en la primera sesión } \\
\text { 3. Inicio de la TREC }\end{array}$ \\
\hline 5 & Individual & $\begin{array}{l}\text { Trabajar sobre Asertividad } \\
\text { Racional Emotivo } \\
\text { Conductual. }\end{array}$ & $\begin{array}{l}\text { Explicación de características de un } \\
\text { comportamiento asertivo desde el punto de } \\
\text { vista de la TREC. }\end{array}$ \\
\hline 6 & Individual & Continuación de la TREC. & Continuación TREC. \\
\hline 7 & Individual & Continuación de la TREC. & Continuación TREC. \\
\hline 8 & Grupal & $\begin{array}{l}\text { Realizar las mediciones } \\
\text { físicas y psicométricas. }\end{array}$ & $\begin{array}{l}\text { 1. Toma de tensión arterial } \\
\text { 2. Aplicación de: } \\
\text { - Cuestionario de Estrés Percibido } \\
\text { - Inventario de Hostilidad de Buss-Durkee } \\
\text { - Inventario de Ansiedad Rasgo-Estado }\end{array}$ \\
\hline
\end{tabular}


Técnicas e instrumentos

La técnica empleada en el presente proyecto de investigación es la terapia o intervención psicológica individual con un total de cuatro pacientes hipertensos del sector. Además, se realizó la aplicación de cuestionarios diseñados para medir las emociones negativas relacionadas con estrés, ira y ansiedad (ver tabla 4). A continuación se describen los principales elementos teóricos de la Terapia Racional Emotivo Conductual según Lega, Caballo y Ellis (2002).

La intervención psicológica se hará con base en los preceptos principales de la Terapia Racional Emotivo Conductual (TREC), cuyo marco de referencia teórico está basado en la premisa de que el pensamiento y las emociones de las personas se yuxtaponen de forma significativa, y que desde el punto de vista práctico, son iguales.

El modelo filosófico general se basa en la premisa de que la perturbación emocional no es creada por las situaciones en sí, sino por las interpretaciones que hacemos de esas situaciones. Además, este modelo terapéutico hace responsable al paciente de su forma de pensar, sentir y comportarse, y no a su pasado o a otras personas.

Si las interpretaciones o creencias (sobre sí mismo, los demás o sobre el mundo en general) son ilógicas, y le dificultan al individuo la obtención de sus metas, entonces son llamadas ideas “irracionales". Según el ABC de este modelo, el método principal para reemplazar una creencia irracional por una racional se llama refutación, cuestionamiento o debate (se cuestionan hipótesis y teorías para determinar su validez, por lo que se compara con el método científico, adaptado a la vida cotidiana).

El modelo ABC de la TREC consta de A, que es el acontecimiento activante (qué sucedió, la realidad confirmable). La B representa la interpretación o evaluación que el paciente realiza del acontecimiento (del inglés belief), que puede ser racional o irracional. Las creencias o cogniciones que las personas tienen pueden ser descriptivas, inferenciales o evaluativas. La C es la consecuencia emocional de la creencia, y es lo que hace que el paciente consulte (se sienten mal, su problema emocional). Hay que diferenciar entre las emociones apropiadas (como serían la preocupación, la tristeza, el enfado, la frustración), y las perturbadoras (como la ansiedad, depresión, ira clínica, culpa y vergüenza).

En este proyecto se emplearon las siguientes herramientas:

- Debate: enseña al paciente a usar el método científico para analizar sus pensamientos irracionales. Una vez identificadas las ideas irracionales, el debate se centra en cuestionar las creencias (la B, del sistema ABC), ya sea a nivel cognitivo, conductual o imaginativo.

- Tareas para la casa: Se le pide al sujeto que practique ciertos ejercicios en casa, entre una sesión y otra. Se asignan al final de cada sesión, y se revisan durante los primeros minutos de la sesión siguiente. Esto permite que se refuerce las habilidades aprendidas durante las sesiones terapéuticas. Además, por medio de las tareas, se facilita el proceso de generalización de lo aprendido en la sesión, a la vida cotidiana.

- Asertividad racional emotivo conductual y resolución de problemas: permite al paciente que aprenda diferentes estrategias para expresar sus sentimientos, actitudes, deseos, opiniones o derechos de una manera adecuada para la situación y contexto en que se encuentra, al respetar esas mismas conductas en los demás (un comportamiento socialmente eficaz). 
- Control de emociones negativas: presenta dos premisas fundamentales al estudiar emociones negativas como la depresión y la ansiedad:

- La rigidez y exigencia absolutista del paciente es la causa principal de la perturbación emocional, y de ella se derivan todo tipo de conclusiones y distorsiones cognitivas.

- Hay dos formas de ansiedad: del ego (relacionada con la depresión) y situacional (más frecuente y menos grave, relacionada con situaciones de incomodidad o baja tolerancia a la frustración.

Tabla 4

Descripción de los cuestionarios diseñados para medir las emociones negativas relacionadas con estrés, ira y ansiedad

\begin{tabular}{|c|c|c|c|}
\hline $\begin{array}{l}\text { Nombre del } \\
\text { instrumento }\end{array}$ & Propósito & Escala de Valoración & Referencia \\
\hline $\begin{array}{l}\text { Cuestionario de } \\
\text { Estrés Percibido }\end{array}$ & $\begin{array}{l}\text { Instrumento autoaplicado } \\
\text { que evalúa el nivel de estrés } \\
\text { percibido durante el último } \\
\text { mes. }\end{array}$ & $\begin{array}{l}\text { Compuesto de } 10 \text { ítems con un } \\
\text { formato de respuesta de una } \\
\text { escala de cinco puntos } \\
\qquad \begin{aligned} 0 & =\text { nunca } \\
1 & =\text { casi nunca } \\
2 & =\text { de vez en cuando } \\
3 & =\text { a menudo } \\
4 & =\text { muy a menudo }\end{aligned}\end{array}$ & $\begin{array}{l}\text { Cohen, Kamarch } \\
\text { y Mermelstein } \\
(1983)\end{array}$ \\
\hline $\begin{array}{l}\text { Inventario de } \\
\text { hostilidad de } \\
\text { Buss-Durkee }\end{array}$ & $\begin{array}{l}\text { Instrumento autoaplicado } \\
\text { diseñado para evaluar la } \\
\text { agresividad. }\end{array}$ & $\begin{array}{l}\text { Compuesto de } 75 \text { afirmaciones, } \\
\text { a las cuales el paciente debe } \\
\text { contestar falso ( } 0 \text { ) o verdadero }(1)\end{array}$ & $\begin{array}{l}\text { Bobes, Portilla, } \\
\text { Bascarán, Sáiz y } \\
\text { Bousoño (2003) }\end{array}$ \\
\hline $\begin{array}{c}\text { Inventario } \\
\text { de Ansiedad } \\
\text { Estado-Rasgo }\end{array}$ & $\begin{array}{l}\text { Instrumento autoaplicado. } \\
\text { Consta de dos partes, una } \\
\text { para determinar el estado de } \\
\text { ansiedad (en un momento } \\
\text { dado), y otra que determina } \\
\text { el rasgo ansioso en la } \\
\text { personalidad. }\end{array}$ & $\begin{array}{l}\text { Compuesto de } 20 \text { frases, y en cada } \\
\text { una el paciente señala cómo la } \\
\text { situación se encuentra en su caso } \\
\text { en este momento: } \\
0=\text { nada } \\
1=\text { algo } \\
2=\text { bastante } \\
3=\text { mucho }\end{array}$ & $\begin{array}{l}\text { Bobes, Portilla, } \\
\text { Bascarán, Sáiz y } \\
\text { Bousoño (2003) }\end{array}$ \\
\hline
\end{tabular}

\section{Resultados}

Se trabajó con un total de participantes hipertensos del EBAIS de San Miguel, que cumplían con los criterios de inclusión propuestos (ver Método). Se tomó la presión arterial de los involucrados en la Sesión 1 y Sesión 8 de trabajo. Los pacientes presentaban una elevación de las cifras de presión arterial en la primera toma. Una vez concluido la intervención psicológica, los pacientes presentaron niveles óptimos o normales de tensión arterial (ver tabla 5). 
Tabla 5

Mediciones de presión arterial antes y después de la intervención psicológica en pacientes hipertensos San Miguel Sarapiquí

\begin{tabular}{cccccc}
\hline Paciente & PA inicial & Clasificación & PA final & Clasificación & Diferencia \\
\hline A.J. & $150 / 100$ & HTA grado 2 & $110 / 75$ & Óptima & $-40 /-25$ \\
M.P. & $140 / 85$ & HTA grado 1 & $130 / 80$ & Normal & $-10 /-5$ \\
S.M. & $140 / 85$ & HTA grado 1 & $105 / 70$ & Óptima & $-35 /-15$ \\
M.S. & $160 / 100$ & HTA grado 2 & $130 / 80$ & Normal & $-30 /-20$ \\
Promedio & $148 / 93$ & - & $119 / 76$ & Óptima & $-29 /-16$ \\
\hline
\end{tabular}

Nota: $\mathrm{PA}=$ presión arterial, HTA = hipertensión.

En cuanto a la valoración de los niveles de estrés, ira y ansiedad, a partir de los tres instrumentos elegidos para el estudio (ver Método) tanto antes como después de la intervención psicológica. La estructura de análisis fue la siguiente:

El Cuestionario de Estrés Percibido (Cohen et al., 1983) tiene una puntuación mínima de 0, y máxima de 40. Los resultados alcanzados por los participantes del estudio fueron categorizados en tres niveles: $0-13=$ leve, $14-26=$ moderado, $27-40=$ severo. Dichos valores fueron convertidos en índices para efectos del análisis.

El Inventario de Ansiedad Estado-Rasgo (Bobes et al., 2003) por otro lado, consta de una puntuación total que oscila entre 0 y 60 . Las puntuaciones directas que se obtienen, se transforman en centiles en función del sexo y la edad, según tabla aportada por los autores. Antes del centil 50, el estado de ansiedad se considera inexistente, entre el centil 50 y el 75 se puede clasificar como leve, entre el centil 75 y el 85 se considera moderado, y entre el centil 85 y el 99 se considera alto.

Adem, el Inventario de Hostilidad de Buss-Durkee indica que desde el punto de vista clínico, posee mayor relevancia el valor cuantitativo de la puntuación total (aunque se proponen diferentes puntos de corte para las subescalas de violencia, hostilidad indirecta, irritabilidad, negativismo, resentimiento, recelos, hostilidad verbal y culpabilidad). El punto de corte de la puntuación total es de 27 puntos.

En términos generales, los participantes del estudio presentaron disminuciones en las mediciones de las tres condiciones investigadas, especialmente en estrés percibido y ansiedad (ver tabla 6). 
Tabla 6

Niveles de estrés percibido, hostilidad (ira) y ansiedad antes y después de la intervención psicológica en pacientes hipertensos San Miguel Sarapiquí

\begin{tabular}{ccccccc}
\hline & \multicolumn{5}{c}{ Mediciones realizadas } \\
\cline { 2 - 6 } Paciente & \multicolumn{2}{c}{ Estrés percibido } & \multicolumn{2}{c}{ Hostilidad (ira) } & \multicolumn{2}{c}{ Ansiedad } \\
\cline { 2 - 6 } & Antes & Después & Antes & Después & Antes & Después \\
\hline A.J. & $58 \%$ & $42 \%$ & 47 & 51 & Alto & Inexistente \\
M.P. & $55 \%$ & $47 \%$ & 46 & 40 & Alto & Moderado \\
S.M. & $45 \%$ & $10 \%$ & 40 & 40 & Bajo (leve) & Inexistente \\
M.S. & $53 \%$ & $27 \%$ & 41 & 34 & Bajo (leve) & Inexistente \\
Promedio & $53 \%$ & $32 \%$ & 44 & 41 & & - \\
\hline
\end{tabular}

\section{Discusión}

Los resultados alcanzados evidencian reducciones en los niveles de tensión arterial, estrés percibido, y ansiedad en todos los pacientes participantes del estudio.

Factores tales como el estrés, ira y ansiedad han sido ligados psicofisiológicamente con la hipertensión arterial, aún así identificar de manera precisa la participación de estos componentes en la alteración de dicha problemática es complejo, ya que los factores emocionales de riesgo para la hipertensión arterial no se presentan aisladamente, sino que se influyen mutuamente, no pudiendose delimitar finamente dónde comienza uno y dónde lo hace otro, y a su vez interactúan con los factores de riesgo tradicional (Fernández et al., 2003).

Por tanto, las investigaciones han intentado establecer relaciones no solo con la incidencia de enfermedad, sino también con la severidad, aún cuando es bastante clara la relación entre estos constructos y los trastornos cardiovasculares, pero se complica cuando se trata de establecer la dirección de esta relación y más si tenemos en cuenta que son constructos multidimensionales. Con el constructo de ira, la mayoría de los estudios apuntan a una dirección clara de la relación entre puntuaciones altas y trastornos coronarios (Fernández \& Martín, 1994).

En cuanto al constructo de ansiedad, se ha planteado que ésta y la aflicción también están relacionadas con trastornos cardíacos, y el estrés predispone a la enfermedad cardiovascular o precipita los episodios isquémicos, ataques cardíacos y otros estados patológicos (Fernández et al., 2003).

Adicionalmente, múltiples estudios han analizado la eficacia que tienen las diferentes técnicas cognitivo-conductuales (entre ellas la Terapia Racional Emotivo Conductual) en la prevención, control y tratamiento de la hipertensión arterial, ya que se ha comprobado la influencia del pensamiento y del comportamiento en el funcionamiento del organismo, y por ende, en su estado de salud.

Molerio y García (2004a) realizaron un estudio exploratorio-descriptivo con un grupo de personas normotensas y otro con pacientes con hipertensión arterial esencial. Se concluyó que éste último está más propenso a vivencias negativas y vulnerabilidad al estrés, así como a depresión, ira y ansiedad. También recomendaron incorporar en estos pacientes programas dirigidos a fomentar estilos de vida saludables, y el desarrollo de habilidades de afrontamiento al estrés y de autocontrol emocional. 
En otro estudio de Molerio y García (2004b) dieron importancia al entrenamiento en técnicas de relajación muscular y técnicas cognitivo-conductuales (como solución de problemas, autocontrol emocional y otras) en el control del paciente hipertenso.

López (2007) realizó una revisión de las técnicas o estrategias metodológicas que se usan en la modificación de conducta para el control, prevención e intervención de la hipertensión arterial. Refiere a la Terapia Racional Emotivo Conductual como eficaz en el tratamiento de pacientes con patrones de comportamiento disfuncionales (como la llamada conducta tipo A), así como en los enfermos coronarios secundarios a éstos.

Miguel et al. (1994), investigadores mexicanos, examinaron los efectos de una intervención cognitivo-conductual en componentes como dieta, actividad física, adherencia al tratamiento farmacológico, estilos de afrontamiento, ansiedad y otros. Encontraron que hubo mejoría clínica estadísticamente significativa en la gran mayoría de estas variables.

Todas estas investigaciones han tenido resultados positivos en cuanto a lograr un mejor manejo del control de los pacientes y de las cifras de tensión arterial que éstos presentan después de las intervenciones, concordando con los resultados ofrecidos en la presente experiencia terapéutica.

\section{Conclusiones}

Este estudio pretendió demostrar la eficacia de la TREC en los niveles de estrés, ira y presión arterial en pacientes hipertensos, los principales hallazgos de la intervención evidencian una disminución en las cifras de tensión arterial posterior a la aplicación de la TREC en los pacientes hipertensos.

Así mismo se infiere que el control de factores como ira, estrés y ansiedad por medio de la TREC ayudó a la reducción de los niveles de presión arterial en los participantes, en concordancia con las conclusiones derivadas en otros estudios.

Aun así debe tenerse claro que la presente propuesta de diseño presenta como limitación la ausencia de un grupo control que potencie los resultados alcanzados, así mismo, el número de sujetos intervenidos es insuficiente para extrapolar de manera general los resultados, lo cual tampoco desmerita los alcances reportados.

Se recomienda la replicación del modelo planteado con un mayor volumen de sujetos y la adhesión de un grupo control que permita aportar mayor validez a los hallazgos del proyecto.

\section{Referencias}

Bobes, J., Portilla, M., Bascarán, M., Sáiz, P., \& Bousoño, M. (2003). Banco de instrumentos básicos para la práctica de la Psiquiatría Clínica. Barcelona: Psiquiatría Editores.

Brannon, L. \& Feist, J. (2001). Psicología de la salud. México: Thomson Editores.

Chapuis, T., Barriguete, J., Cheron, P., Hernández, M., Vieyra, A., \& García-Viveros, M. (agosto, 2006). Hipertensión arterial: diagnóstico y tratamiento. Boletín Práctica Médica Efectiva (4)8, Recuperado de http://bvs.insp.mx/articulos/1/13/v4n8.pdf

Cohen, S., Kamarck, T., \& Mermelstein, R. (1983). A global measure of perceived stress. Journal of Health and Social Behavior, 24, 385-396.

DiMatteo, M. R. (marzo, 2004). Social support and patient adherence to medical treatment: a meta-analysis. Health Psychology, 23(2), 207-218. doi: 10.1037/0278-6133.23.2.207

Fernández, E. G. \& Martín, M. D. (1994). Ira y hostilidad en los trastornos coronarios. Anales de Psicología, 10(2), 177-188. 
Fernández, E. G., Martín, M. D., \& Domínguez, F. (2003) Factores de riesgo e intervenciones psicológicas eficaces en los trastornos cardiovasculares. Psicothema, 15(4), 615-630.

Friedman, R., Schwartz, J. E., Schall, P. L., Pieper, C., Gerin, W., \& Pickering, T. G. (2001). Psychological variables in hypertension. Relationships to casual or ambulatory blood pressure in men. Psychosomatic Medicine, 63, 19-31.

Hernández, R., Fernández, C., \& Baptista, L. (2006). Metodología de la investigación. México: Mcgraw Hill.

Jiménez, E. (2002). Manual para la detección, diagnóstico y tratamiento de la hipertensión arterial en el primer nivel de atención. Caja Costarricense de Seguro Social. Recuperado de http://www.binasss.sa.cr/hipertension.pdf.

Jorgensen, R. S., Johnson, B. T., Kolodziej, M. E., \& Schreer, G. E. (septiembre, 1996). Elevated blood pressure and personality: a meta-analytic review. Psychological Bulletin, 120(2), 293-320. doi: 10.1037/0033-2909.120.2.293

Labiano,L., Brusasca, C., \& Gargani, A. (2005). Control de la presión arterial a través de técnicas de relajación. Manual de Psicología Clínica y de Salud Hospitalaria. Colombia: Psicom Editores.

Lega, L., Caballo, V., \& Ellis, A. (2002). Teoría y práctica de la terapia racional emotivoconductual. España: Ediciones Siglo XXI.

López González, F. J. (mayo, 2007). Prevención, técnicas de modificación de conducta para el tratamiento de la hipertensión arterial (HTA) y otros factores de riesgo. Revista Electrónica Psicología Cientifica. Recuperado de www.psicologiacientifica.com/bv/psicologia-275.

Miguel-Tobal, J. J., Cano-Vindel, A., Casado-Morales, M. I., \& Escalona-Martínez, A. (1994). Emociones e hipertensión. Implantación de un programa cognitivo-conductual en pacientes hipertensos. Anales de Psicología, 10(2), 199-216.

Molerio Pérez, O. \& García Romagosa, G. (2004a). Contribuciones y retos de la psicología de la salud en el abordaje de la hipertensión arterial esencial. Revista de Psicología, 13(2), 101-109.

Molerio Pérez, O. \& García Romagosa, G. (abril-junio, 2004b). Influencia del estrés y las emociones en la hipertensión arterial esencial. Revista Cubana de Medicina, 43(2-3).

Sommers, J. \& Greenberg, R. P. (enero, 1989). Psychological variables and hypertension: a new look at an old controversy. Journal of Nervous and Mental Disease, 177(1), 15-24. doi: 10.1097/00005053-198901000-00003

Vögele, C. \& Steptoe, A. (1992). Emotional coping and tonic blood pressure as determinants of cardiovascular responses to mental stress. Journal of Hypertension, 10(9), 1079-1087.

\section{Reseña de los autores}

\section{Federico Holst Schumacher}

Licenciado en Medicina por la Universidad de Costa Rica y Licenciado en Psicología por la Universidad de Iberoamérica. Labora como Médico en la Caja Costarricense de Seguro Social Área de Salud de Aguas Zarcas, San Carlos.

\section{Diego Quirós Morales}

Licenciado en Psicología por la Universidad Católica de Costa Rica. Labora como Investigador para la Fundación Omar Dengo. 